\title{
重金属 $\mathrm{Zn}^{2+}$ 胁迫诱导斜纹夜蛾幼虫血细胞调亡
}

\author{
夏 嫱 孙虹霞 胡新军 舒迎花 古德祥 张古忍*
}

(中山大学有害生物控制与资源利用国家重点实验室, 昆虫学研究所, 广州 510275.**联系人, E-mail: zhanggr@mail.sysu.edu.cn)

\begin{abstract}
摘要 通过在人工饲料中添加不同浓度的 $\mathrm{Zn}^{2+}$, 采用流式细胞技术, 初步分析了植食性昆虫斜纹夜蛾 Spodoptera litura Fabricius 幼虫因过量摄入 $\mathrm{Zn}^{2+}$ 而诱发的血细胞调亡. 结果表明, 饲料中 $\mathrm{Zn}^{2+}$ 浓度的增 加会导致幼虫血淋巴和脂肪体中 $\mathrm{Zn}^{2+}$ 的积累, 二者之间表现出显著的剂量-反应关系, 且脂肪体对 $\mathrm{Zn}^{2+}$ 的累积强度明显高于血淋巴; 当 $\mathrm{Zn}^{2+}$ 浓度达到 $1000 \mathrm{mg} / \mathrm{kg}$ 时, 能显著诱导血细胞的调亡, 调亡率为 $63.63 \%$ ，显著高于对照和低浓度(50 500 mg/kg)胁迫而产生的调亡率. 因此, 食物中高浓度的 $\mathrm{Zn}^{2+}$ 能诱 导植食性昆虫斜纹夜蛾幼虫血细胞的调亡。
\end{abstract}

\section{关键词 重金属 $\mathrm{Zn}^{2+}$ 斜纹夜蛾 细胞调亡}

细胞凋亡是细胞受到内、外因子刺激后发生的 由自身基因调控的生理性死亡行为, 是一个主动和 高度有序的生命活动过程 ${ }^{[1.2]}$, 其作用在于清除体内 多余的或损伤的细胞 ${ }^{[3]}$. 在众多的调亡诱导因子中, 重金属是认识较早的典型诱导细胞调亡因子之一 44.5$]$. 正常情况下, 重金属(如 $\mathrm{Zn}$ 等)对生物体的各种生命活 动具有独特的生理效应, 能调节细胞的生长发育, 但随着工业化和城市化的发展, 重金属又成为最严 重的环境污染因子之一, 严重影响了有机体正常的 生理生化代谢和遗传发育, 甚至诱发癌变. 特别是 近年来发现镉、铬、镍、铅、砷、采、铜和锌等均 可诱导或影响细胞调亡 ${ }^{[6 \sim 9]}$, 如过量摄人锌可引起小 鼠生精细胞的调亡 ${ }^{[10]}$.

重金属污染已成为世界性的环境问题, 全球每 年向环境中排放锌量多达 1372000 吨 ${ }^{[11]}$. 我国也不 例外, 如珠江三角洲农田表层土中的锌含量平均为 $166.9 \mathrm{mg} / \mathrm{kg}$, 最高达到 $1343 \mathrm{mg} / \mathrm{kg}$, 而背景值仅为 $49.7 \mathrm{mg} / \mathrm{kg}^{[12]}$. 在富锌土壤中种植的十字花科蔬菜, 收获期菜叶中锌含量高达 $1297.5 \mathrm{mg} / \mathrm{kg}$, 花苔含量达 $1141.8 \mathrm{mg} / \mathrm{kg}$, 其他蔬菜也有不同程度的富集作用 ${ }^{[13]}$. 毫无疑问, 植食性昆虫可能因此而受到一定影响 ${ }^{[14]}$.

斜纹夜蛾 Spodoptera litura Fabricius(鳞翅目, 夜 蛾科)是一种杂食性害虫, 其寄主范围极其广泛, 可 为害烟草等 99 科 290 多种植物 ${ }^{[15]}$. 基于其独特的食 性特点, 日益严重的环境污染是否会对其行为、生 理、遗传变异、种群暴发等产生一定的影响呢? 这是 我们目前应该关注与有待研究的问题.

流式细胞技术是目前世界上最先进的细胞定量 分析技术, 是一种利用光散射、苂光辐射和吸收对高
速流动的细胞或亚细胞进行快速定量测定和分析的 方法. 它每秒钟能分析几千个细胞, 并同时测定细 胞的多个参数, 如细胞的特征(形态、膜电位等)和细 胞内蛋白质含量、表面受体、DNA 及 $\mathrm{pH}$ 等, 已成为 生物学、药理学、毒理学、细菌学、病毒学、环境 科学和生物技术研究的有用工具 ${ }^{[16]}$. 本研究将这一 技术引人昆虫学研究领域, 通过在人工饲料中添加 不同浓度 $\mathrm{Zn}^{2+}$ 来胁迫斜纹夜蛾幼虫, 在测定幼虫血 淋巴、脂肪体 $\mathrm{Zn}^{2+}$ 含量的基础上, 初步分析了不同浓 度 $\mathrm{Zn}^{2+}$ 胁迫对斜纹夜蛾幼虫血细胞调亡的诱导作用, 以期揭示农田重金属污染对植食性昆虫可能产生的 潜在影响.

\section{1 材料与方法}

(i) 斜纹夜蛾幼虫的饲养. 斜纹夜蛾系中山大 学昆虫学研究所养虫室人工饲料饲养 ${ }^{[17]}$. 在人工饲料 中添加不同浓度的 $\mathrm{Zn}^{2+}$, 并以此连续 3 代饲喂斜纹夜 蛾幼虫: 以第 1 代幼虫化蛹后, 羽化成虫所产的卵作为 第 2 代虫源, 第 3 代以此类推. 以第 3 代 4 龄幼虫作为 测试材料, 不添加 $\mathrm{Zn}^{2+}$ 的饲料为对照. 所有的昆虫均 饲养在 $(26 \pm 1)^{\circ} \mathrm{C}, 16 \mathrm{~L} ： 8 \mathrm{D}, \mathrm{RH}(75 \pm 5) \%$ 条件下.

(ii) 仪器与试剂. FAC Svantage SE 型流式细 胞仪为 Becton Dickinson(BD)公司生产; IRIS Advantage (HR) 等离子体原子发射光谱仪由美国 Thermo Jarrell Ash Corporration 生产. 调亡细胞 DNA 苂光染 料 Hoechst 33258 和死亡细胞 DNA 染料碘化丙啶 (Propidium Iodide, PI)均购自 Sigma 公司.

生理盐水为鳞翅目昆虫专用, 主要成分为: $\mathrm{NaCl}$, $7.0 \mathrm{~g} / \mathrm{L} ; \mathrm{KCl}, 0.2 \mathrm{~g} / \mathrm{L} ; \mathrm{CaCl}_{2}, 0.1 \mathrm{~g} / \mathrm{L} ; \mathrm{MgCl}_{2}, 0.1 \mathrm{~g} / \mathrm{L} ;$ $\mathrm{NaHCO}_{3}, 0.15 \mathrm{~g} / \mathrm{L} ; \mathrm{NaH}_{2} \mathrm{PO}_{4}, 0.2 \mathrm{~g} / \mathrm{L} ; \mathrm{C}_{6} \mathrm{H}_{6} \mathrm{O}_{6}, 7.0$ 
$g / L^{[18]}$. 所有无机试剂均为广州试剂一厂生产的分析 纯试剂.

(iii) $\mathrm{Zn}^{2+}$ 的胁迫处理. 重金属 $\mathrm{Zn}^{2+}$ 来源于 $\mathrm{ZnCl}_{2}$, 将 $\mathrm{ZnCl}_{2}$ 添加在人工饲料中, 使 $\mathrm{Zn}^{2+}$ 终浓度分 别为 $50,100,150,500$ 和 $1000 \mathrm{mg} / \mathrm{kg}$. 以不添加 $\mathrm{ZnCl}_{2}$ 饲料饲养的斜纹夜蛾幼虫为对照.

(iv) 幼虫脂肪体和血淋巴对 $\mathrm{Zn}^{2+}$ 的积累. 取 30 头第 3 代 4 龄幼虫, 用昆虫针刺第 3 腹足取其血 淋巴后, 解剖并收集体腔内脂肪体. 将脂肪体放人 $120^{\circ} \mathrm{C}$ 烘箱干燥 $4 \mathrm{~h}$ 后, 立即放人干燥器中冷却. 然 后称量 $0.1 \mathrm{~g}$ 干燥脂肪体与 $2 \mathrm{~mL}$ 血淋巴分别进行消 化. 先将材料放人 $50 \mathrm{~mL}$ 安培瓶中, 再加人 $10 \mathrm{~mL}$ 浓硝酸, 置于电炉上消化. 待棕色气体消失冒白烟 后移开安培瓶, 自然冷却后加人 $1 \mathrm{~mL}$ 浓高氯酸继续 消化. 待棕色气体消失, 得到透明溶液后取下安培 瓶, 冷却后用定性滤纸过滤, 去离子水定容至 $50 \mathrm{~mL}$. 以浓硝酸和高氯酸为空白对照, 用等离子体原子发 射光谱仪测定各个样品的 $\mathrm{Zn}^{2+}$ 含量, 每个处理 3 个重复.

（V）幼虫血细胞的凋亡. 用昆虫针刺第 3 代 4 龄幼虫第 3 腹足取血淋巴, 将血淋巴滴在 parafilm上, 每个处理 3 个重复(每个重复为 10 头幼虫). 每个重复 各取 $20 \mu \mathrm{L}$ 血淋巴, 分别加在含有 $30 \mu \mathrm{L}$ 生理盐水的 $1.5 \mathrm{~mL}$ 离心管(EP)中, 离心取血细胞, 在血细胞中分 别加人终浓度为 $10 \mu \mathrm{g} / \mathrm{mL}$ 的 Hoechst33258 苂光染 料 $5 \mu \mathrm{L}, 37^{\circ} \mathrm{C}$ 水浴恒温箱中反应 $10 \mathrm{~min}$. 取出后立即 加人终浓度为 $50 \mu \mathrm{g} / \mathrm{mL}$ PI 染料 $5 \mu \mathrm{L}$, 避光、冰上反 应 $5 \mathrm{~min}$, 加生理盐水至 $500 \mu \mathrm{L}$. 用滤膜过滤到 FACS 管中待测, PI 反应后的各步骤均在冰上进行. 以正常未处理未染色细胞为空白对照, 以正常未处 理染色细胞为对照.

对染色后的细胞采用流式细胞仪进行分析. Hoechst 用紫外多谱激发, 功率为 $500 \mathrm{mV}$, PI 用 488 $\mathrm{nm}$ 氦激光激发, 功率为 $150 \mathrm{mV}$. 每个样本检测 10000 个细胞, 以细胞调亡的百分率为观测指标.

( vi ) 数据分析. 组织中的 $\mathrm{Zn}^{2+}$ 浓度数据经 $\log _{10}(x+1)$ 转换, 血细胞调亡率、死亡率、机械损伤率 和存活率数据经 $\arcsin$ 转换后, 采用 SAS 软件包 ${ }^{[19]}$ 进 行方差分析, 并进行多重比较, 以阐明对照与处理间 以及各个处理间差异的显著性. 对脂肪体和血淋巴中 的重金属含量与斜纹夜蛾幼虫饲料中的 $\mathrm{Zn}^{2+}$ 浓度进行 线性回归分析, 以了解组织中的含量与饲料中离子浓 度之间的关系。

\section{2 结果}

\section{1 斜纹夜蛾幼虫脂肪体和血淋巴对 $\mathrm{Zn}^{2+}$ 的积累}

斜纹夜蛾幼虫经 $\mathrm{Zn}^{2+}$ 连续胁迫 3 代后, 第 3 代 4 龄幼虫血淋巴和脂肪体的 $\mathrm{Zn}^{2+}$ 含量随着饲料中 $\mathrm{Zn}^{2+}$ 浓度的增加而增加, 均与处理浓度显著相关(脂肪体, $R^{2}=0.9412, P<0.05$; 血淋巴, $R^{2}=0.8991, P<0.05$ ), 呈现出明显的剂量-反应关系, 且脂肪体对 $\mathrm{Zn}^{2+}$ 的积 累强度明显高于血淋巴(图 1). 当饲料中 $\mathrm{Zn}^{2+}$ 浓度分 别为 50 和 $100 \mathrm{mg} / \mathrm{kg}$ 时, 脂肪体中 $\mathrm{Zn}^{2+}$ 的含量分别 为 $60.42 \pm 9.96$ 和 $71.42 \pm 2.96 \mathrm{mg} / \mathrm{kg}$, 极显著低于饲料 中 $\mathrm{Zn}^{2+}$ 浓度为 150,500 和 $1000 \mathrm{mg} / \mathrm{kg}$ 时脂肪体中 $\mathrm{Zn}^{2+}$ 的含量 $(144.42 \pm 6.86,170.50 \pm 6.86$ 和 $360.67 \pm 8.38$ $\mathrm{mg} / \mathrm{kg}$ ), 而后三者相互间又差异极显著 $(\mathrm{d} f=12, F=$ $297.61, P<0.01)$. 当饲料中 $\mathrm{Zn}^{2+}$ 浓度为 100,150 和 $500 \mathrm{mg} / \mathrm{kg}$ 时, 血淋巴中 $\mathrm{Zn}^{2+}$ 的含量分别为 40.62 $\pm 1.37,43.33 \pm 2.17$ 和 $47.67 \pm 2.33 \mathrm{mg} / \mathrm{kg}$, 其差异不显 著, 但极显著高于饲料中 $\mathrm{Zn}^{2+}$ 浓度为 $50 \mathrm{mg} / \mathrm{kg}$ 时血 淋巴的 $\mathrm{Zn}^{2+}$ 含量 $(25.08 \pm 2.53 \mathrm{mg} / \mathrm{kg})$, 又极显著低于 饲料中 $\mathrm{Zn}^{2+}$ 浓度为 $1000 \mathrm{mg} / \mathrm{kg}$ 时血淋巴的 $\mathrm{Zn}^{2+}$ 含量 $(82.42 \pm 2.46 \mathrm{mg} / \mathrm{kg})(\mathrm{d} f=12, F=91.14, P<0.01)$. 因 此, 当斜纹夜蛾幼虫食料中 $\mathrm{Zn}^{2+}$ 含量增加时, 会导致 幼虫血淋巴和脂肪体中出现 $\mathrm{Zn}^{2+}$ 的累积.

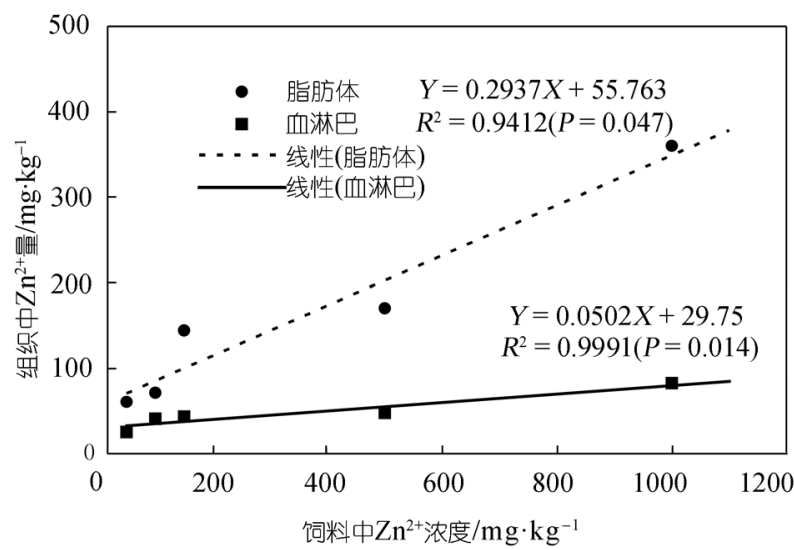

图 1 斜纹夜蛾幼虫脂肪体和血淋巴对 $\mathrm{Zn}^{2+}$ 的积累

\section{2 幼虫血细胞的调亡}

正常斜纹夜蛾幼虫和各处理幼虫血细胞的流式 细胞分析结果见图 2, 与之对应的各处理组幼虫血细 胞的调亡率、死亡率和存活率见表 1. 检测结果表明, 斜纹夜蛾幼虫血细胞的调亡率、死亡率和存活率在 $\mathrm{Zn}^{2+}$ 处理浓度为 $0,50,150,500 \mathrm{mg} / \mathrm{kg}$ 时差异不显著; 但处理浓度达到 $1000 \mathrm{mg} / \mathrm{kg}$ 时, 斜纹夜蛾幼虫血细 
表 1 斜纹夜蛾幼虫取食含不同 $\mathrm{Zn}^{2+}$ 的食料后对血细胞的调亡率 ${ }^{\mathrm{a})}$

\begin{tabular}{cccc}
\hline $\mathrm{Zn}^{2+}$ 浓度 $/ \mathrm{mg}^{\mathrm{kg}} \mathrm{kg}^{-1}$ & 调亡率 $(\%)$ & $8.06 \pm 1.818 \mathrm{~b}$ & 存活率 $(\%)$ \\
\hline 0 (对照 $)$ & $0.28 \pm 0.159 \mathrm{~b})$ & $94.41 \pm 2.372 \mathrm{~b}$ \\
50 & $2.70 \pm 0.556 \mathrm{~b}$ & $9.02 \pm 3.553 \mathrm{~b}$ & $84.09 \pm 3.497 \mathrm{~b}$ \\
100 & $1.28 \pm 0.112 \mathrm{~b}$ & $4.01 \pm 0.144 \mathrm{~b}$ & $79.65 \pm 2.758 \mathrm{~b}$ \\
150 & $0.74 \pm 0.188 \mathrm{~b}$ & $5.87 \pm 1.509 \mathrm{~b}$ & $85.02 \pm 1.801 \mathrm{~b}$ \\
500 & $0.26 \pm 0.211 \mathrm{~b}$ & $10.37 \pm 2.465 \mathrm{~b}$ & $83.76 \pm 4.497 \mathrm{~b}$ \\
1000 & $63.63 \pm 12.623 \mathrm{a}$ & $31.56 \pm 11.340 \mathrm{a}$ & $3.13 \pm 0.681 \mathrm{a}$ \\
\hline
\end{tabular}

A) 同一列平均数后不同的小写字母表示差异显著. 调亡率: $\mathrm{d} f=12, F=24.54, P<0.01$; 死亡率: $\mathrm{d} f=12, F=3.96, P<0.05 ;$ 存活率: $\mathrm{d} f=$ $12, F=130.85, P<0.01$.
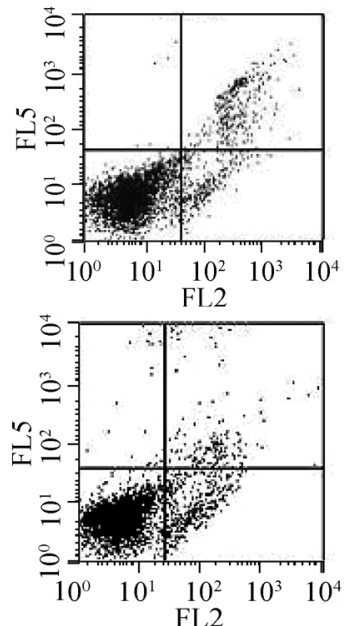

(a)

(d)

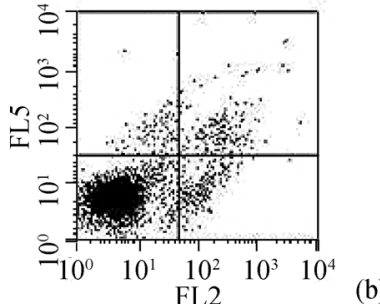

(b)

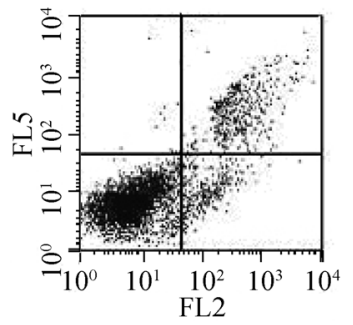

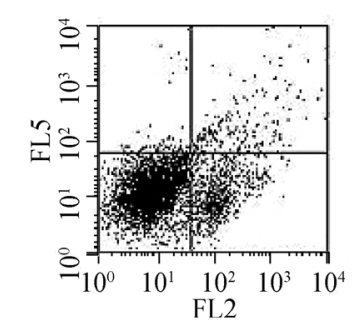

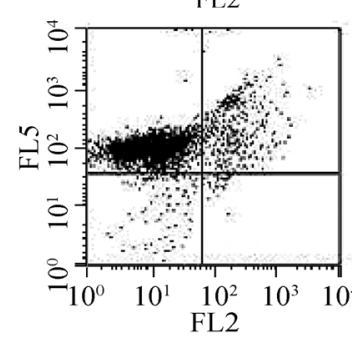

)

(c)

(e)

图 2 不同浓度 $\mathrm{Zn}^{2+}$ 胁迫下斜纹夜蛾幼虫血细胞的流式细胞分析图

(a) 对照; (b) $50 \mathrm{mg} / \mathrm{kg}$; (c) $100 \mathrm{mg} / \mathrm{kg}$; (d) $150 \mathrm{mg} / \mathrm{kg}$; (e) $500 \mathrm{mg} / \mathrm{kg}$; (f) $1000 \mathrm{mg} / \mathrm{kg}$. $X$ 轴为 PI 染色细胞数; $Y$ 轴为 Hoechst33258 染色细胞数. 左上格为调亡细胞数; 右上格为死亡细胞数; 左下格为正常细胞数; 右下格为机械损伤细胞数

胞的调亡数占全体细胞数的 $63.63 \%$, 极显著高于对 照和其他浓度 $\mathrm{Zn}^{2+}$ 处理下的调亡率, 死亡率也显著 高于对照和其他处理, 细胞存活率则仅为全体细胞 数的 $3.13 \%$, 极显著低于对照和其他浓度处理的细胞 存活率. 因此, 当斜纹夜蛾幼虫食料中的 $\mathrm{Zn}^{2+}$ 含量增 加到一定程度后能诱导幼虫血细胞的调亡.

\section{3 讨论}

锌是动物体营养所必需的微量元素之一. 已经 证明, 锌参与动物体内 300 余种酶和功能蛋白的组 成, 具有广泛的生理生化功能. 因此, 动物食料中适 量的锌对维持机体的正常生理代谢和生长发育具有 重要的意义 ${ }^{[20]}$.

本研究表明, $\mathrm{Zn}^{2+}$ 可以在斜纹夜蛾幼虫的血细胞 和脂肪体中积累, 并对幼虫血细胞产生一定的影响. 当斜纹夜蛾幼虫饲料中 $\mathrm{Zn}^{2+}$ 浓度增加到一定程度后, 幼虫血淋巴和脂肪体中 $\mathrm{Zn}^{2+}$ 的积累量会显著升高, 并随着处理浓度增大而增加, 呈现出明显的剂量-反 应关系, 且脂肪体中 $\mathrm{Zn}^{2+}$ 含量显著高于血淋巴. 这种 作用可能和特定组织的结构和功能有密切的联系.
Brown $^{[21]}$ 和 Hopkin ${ }^{[22]}$ 的研究表明, 陆生和水生节肢 动物对过量重金属的解毒机制有明显的不同. 陆生 节肢动物不能像水生节肢动物那样直接通过呼吸作 用将过量的重金属经由血淋巴排出体外，而是将过 量的重金属以颗粒的形式沉积在具有消化、储存或 分泌功能的器官, 而且这种机制也适用于昆虫等消 化系统具有单层细胞的生物. 此外, 在一些其他无 脊椎动物中也有类似的现象, 如水体中的重金属首 先进人紫贻贝 Mytilus edulis (L)的血淋巴, 然后再转 运到其他部位进行解毒 ${ }^{[23]}$. 在斜纹夜蛾幼虫中, 血 淋巴也可能具有转运作用, 大量 $\mathrm{Zn}^{2+}$ 被转运到脂肪 体进行解毒, 从而降低了重金属的危害. 类似的是, 唇足纲的 Lithobius variegates (Leach)也将过量的 $\mathrm{Zn}^{2+}$ 贮存在脂肪体中 ${ }^{[24]}$, 蜻蜓的稚虫、蚂蚁幼虫以及 蝗蝻等的脂肪体也能咜存多余的重金属 ${ }^{[25,26]}$.

锌在体内主要以 $\mathrm{Zn}^{2+}$ 以及锌依赖酶或其他锌蛋 白的形式存在, 参与基因的复制转录和蛋白质的生 物合成、激素与受体的特异性结合以及信号转导等. 锌对细胞凋亡的影响强度主要取决于细胞内的锌含 量和作用时间. 因此, $\mathrm{Zn}^{2+}$ 对细胞凋亡的影响没有表 
现出如血淋巴中 $\mathrm{Zn}^{2+}$ 含量那样明显的剂量-反应关系. 只有当饲料中 $\mathrm{Zn}^{2+}$ 达到一定的浓度, 如 $1000 \mathrm{mg} / \mathrm{kg}$ 时, 血淋巴中的 $\mathrm{Zn}^{2+}$ 大量积累时诱导了血细胞的调 亡. 但红䲡鱼 Oncorhynchus mykiss (Walbaum)上皮细 胞的调亡率随 $\mathrm{Zn}^{2+}$ 浓度的增加而增加, 二者间存在 一定的剂量反应关系 ${ }^{1)}$; 牡蚛 Crassostrea virginica (Gmelin)血细胞调亡与 $\mathrm{Cd}^{2+}$ 浓度之间存在明显的剂 量-反应关系 277 . 这可能是由于不同重金属的特性以 及作用种属特异性的原因. 此外, Bischof ${ }^{\mathrm{f} 28}$ 对森林 害虫舞毒蛾 Lymantria dispar L 的研究表明, $\mathrm{Zn}^{2+}$ 能影 响舞毒蛾幼虫血淋巴中的糖类和脂类代谢, 降低血 淋巴中茧蜜糖(trehalose)和山梨[糖]醇(sorbitol)的含量. 因此, 斜纹夜蛾幼虫摄人过量锌后, 能诱导血细胞 的大量调亡, 从而对其正常的生长发育和生理机能 产生一定的影响, 但其机理尚需要进一步研究.

在野外条件下，富锌土壤会导致蔬菜等作物对 锌的奢侈吸收 ${ }^{[13]}$, 作为斜纹夜蛾幼虫主要寄主植物 的十字花科蔬菜, 其叶和花苔中锌含量可分别高达 1297.5 和 $1141.8 \mathrm{mg} / \mathrm{kg}$, 均超过本研究所设置的最高 处理浓度 $(1000 \mathrm{mg} / \mathrm{kg})$. 因此, 本研究结果可以在一 定程度上揭示, 重金属锌引起的农田土壤污染, 能 够通过植食性昆虫的寄主植物间接地对植食性昆虫 产生一定的影响, 如诱导血细胞调亡等.

关于重金属 $\mathrm{Zn}^{2+}$ 胁迫对斜纹夜蛾的全面影响尚 需要进一步研究, 包括对昆虫行为、生理和种群增殖, 以及昆虫因此而产生的适应等各个方面.

致谢 本工作为广东省科技计划(批准号：2005B20501005) 和广东省自然科学基金(批准号: E039254)资助项目。

\section{参考文献}

1 Kerr J F, Wyllie A H, Currie A R. Apoptosis: A basic biological phenomenon with wide-ranging implications in tissue kinetics. $\mathrm{Br}$ J Cancer, 1972, 26(4): 239 257

2 刘欣梅, 项黎新, 邵健忠, 等. 重金属诱导细胞调亡的分子机 制. 细胞生物学杂志, 2004, 26(3): 235 240

3 Hay B A, Huh J R, Guo M. The genetics of cell death: Approaches, insights and opportunities in Drosophila. Nat Rev, 2004, 5: 911 922[DOI]

4 Wolfe J T, Ross D, Cohen G M. A role for metals and free radicals in the induction of apoptosis in thymocytes. FEBS Lett, 1994, 352: 58 62[DOI]

5 Rajaram R, Nair B U, Ramasami T. Chromium III -induced abnormalities in human lymphocytes cell proliferation: Evidence for apoptosis. Biochem Biophys Res Commun, 1995, 210: 434 440[DOI]

6 Habbeebu S S, Liu J, Klaassen C D. Cadmium-induced apoptosis in mouse liver. Toxicol Appl Pharmacol, 1998, 149(2): 203 209[DOI]

7 Lee S H, Kim D K, Seo Y R, et al. Nickel( II )-induced apoptosis and G2/M enrichment. Exp Mol Med, 1998, 30: 171 176

8 Lohmann R, Beyersmann D. Cadium and zinc mediated changes of the $\mathrm{Ca}^{2+}$-dependent endonuclease in apoptosis. Biochem Biophys Res Comm, 1993, 190: 1097 1103[DOI]

9 Raes H, Braekman, B P, Criel G R J, et al. Copper induces apoptosis in Aedes C6/36 cells. J Exp Zool, 2000, 286: 1 12[DOI]

10 刘晋芝, 张晶晶, 王玉庆, 等. 过量摄人锌引起生精细胞调亡 的实验研究. 军医进修学院学报, 2005, 26(3): 240

11 Kovalchuk O, Titov V, Hohn B, et al. A sensitive transgenic plant system to detect toxic inorganic compounds in the environment. Nature Biotech, 2001, 19: 568 572[DOI]

12 柴世伟，温琰茂，韦献革，等. 珠江三角洲主要城市郊区农业 土壤的重金属含量特征. 中山大学学报 (自然科学版), 2004, 43(4): 90 94

13 吴金桂, 李正明, 徐跃定, 等. 蔬菜奢侈吸锌特性研究. 土壤通 报, 1996, 27(5): 228 229

14 Mulder C, Aldenberg T, de Zwart D, et al. Evaluating the impact of pollution on plant-Lepidoptera relationships. Environmetrics, 2005, 16: 357 373[DOI]

15 陈乾锦, 张根顺, 官宝斌, 等. 烟田斜纹夜蛾侧沟茧蜂的生物 学和生态学研究. 江西农业大学学报, 2003, 25(2): 199 203

16 谢小梅, 许杨. 流式细胞技术. 中国生物工程杂质, 2003, 23(9): 100 104

17 陈其津, 李广宏, 庞义. 饲养 5 种夜蛾科昆虫的一种简易人工 饲料. 昆虫知识, 2000, 37(6): 325 327

18 叶恭银, 胡萃, 吴生泉. 天虫雄性内生殖器官的结构及其发育 观察. 浙江农业学报, 1996, 8(6): 368 371

19 SAS Institute Inc. SAS/STAT User's Guider. Ver 6, 4th ed. Cary, NC: SAS Institute Inc, 1989. Vol 1 and 2

20 井明艳, 孙建义, 赵树盛, 等. 微量元素锌的营养学研究进展. 饲料工业, 2004, 25(10): 20 24

21 Brown B E. The form and function of metal-containing 'granules' in invertebrate tissues. Biol Rev, 1982, 57: 621 667

22 Hopkin S P. Critical concentrations, pathways of detoxification and cellular ecotoxicology of metals in terrestrial arthropods. Func Ecol, 1990, 4: 321 327

23 Renwrantz L, Schmalmack W, Steenbuck M. Molecular size of native proteins of Mytilus serum which contains a dominant frac tion with heavy metal-binding properties. Comp Biochem Physiol, Part A: Physiol, 1998, 121: 175 180

24 Hopkin S P, Martin M H. Heavy metals in the centipede Lithobius variegates (Chilopoda). Environ Pollut, Series B: Chem Phys, 1983, 6: 309 318

25 Meyer W, Harisch G, Sagredos A N. Biochemical and histo chemical aspects of lead exposure in dragonfly larvae (Odonata: Anisoptera). Ecotoxicol Environ Saf, 1986, 11: 308 319[DOI]

26 Ballan-Dufrancais C. Localization of metals in cells of Pterygote insects. Micros Res Tech, 2002, 56: 403 420[DOI]

27 Sokolova M I, Evans S, Hughes M. Cadmium-induced apoptosis in oyster hemocytes involves disturbance of cellular energy bal ance but no mitochondrial permeability transition. J Exp Biol, 2004, 207: 3369 3380 [DOI]

28 Bischof C. Effects of heavy metal stress on carbohydrate and lipid concentrations in the haemolymph and total body tissue of parasi tized Lymantria dispar L. larvae (Lepidoptera). Comp Biochem Physiol, 1995, 112C(1): 87 92

(2005-08-26 收稿, 2005-10-21 收修改稿)

1) Shúilleabháin S N, Mothersill C, Sheehan D, et al. Cellular responses in primary epidermal cultures from rainbow trout exposed to zinc chloride. Ecotoxicol Environ Saf, 2005 (待发表) 